\title{
Differentiation by random amplified polymorphic DNA-polymerase chain reaction (RAPD-PCR) of Candida albicans isolated from upper respiratory tract in patients with non-small cell lung cancer*
}

\author{
Anna Biernasiuk ${ }^{\bowtie}$, Izabela Korona-Głowniak, Agnieszka Grzegorczyk and Anna Malm \\ Department of Pharmaceutical Microbiology, Medical University of Lublin, Lublin, Poland
}

Cancer patients are predisposed to fungal infections caused by Candida albicans, especially to oral or respiratory tract candidiasis. The aim of this study was to estimate genetic diversity by RAPD-PCR (random amplified polymorphic DNA-polymerase chain reaction) of C. albicans isolated from upper respiratory tract of 100 patients with non-small cell lung cancer. Among 52 strains, 34 genotypes were defined. 10 clusters comprising 28 $(53.85 \%)$ isolates with similarity coefficient $\geq 80 \%$ were formed. The remaining $24(46.15 \%)$ isolates represented individual genotypes. The RAPD-PCR technique revealed genomic variability within $C$. albicans isolated from upper respiratory tract of the cancer patients.

Key words: RAPD-PCR, Candida albicans, upper respiratory tract, genetic diversity

Received: 29 November, 2013; revised: 12 June, 2014; accepted:

17 October, 2014; available on-line: 06 November, 2014

\section{INTRODUCTION}

Yeasts belonging to Candida spp., mainly Candida albicans, are commensal organisms that can be isolated from the gastrointestinal tract, as well as oral and vaginal mucosa of many, if not all, healthy individuals (Kim \& Sudbery, 2011). At the same time, C. albicans is one of the most common opportunistic pathogens which can cause endogenous infections from superficial to seriously deepseated mycoses under altered host conditions (Chong et al., 2003; Pires-Gonçalves et al., 2007; Costa et al., 2008; Bacelo et al., 2010; Gültekin et al., 2011).

The number of infections caused by these microorganisms has grown rapidly in the last years and they are an important medical problem in patients from high risk groups, such as immunocompromised people or malignant cancer patients. Anticancer treatment including chemotherapy and/or radiotherapy is still associated with several long-term adverse effects. Among them, oral candidiasis is one of the major sources of illness, although strict oral hygienic care by using antifungal reagents is being employed to prevent it (Teanpaisan et al., 2008; Sun et al., 2009; Lee et al., 2010).

Cancer patients, among them patients with nonsmall cell lung cancer, are also predisposed to infections caused by $C$. albicans, especially oral or respiratory tract candidiasis. Such infections are usually endogenous in origin due to colonization of the upper respiratory tract mucosa by the yeast. There are many important risk fac- tors predisposing patients to such colonization, including for e.g.: long-term anticancer therapy (chemotherapy and/or radiotherapy), long hospital stay, surgical procedures, intravenous drug administration or endocrynological disorders (Chong et al., 2003; Samaranayake et al., 2003; Costa et al., 2008).

Since the colonizing and invading Candida sp. isolates are usually the same, actual knowledge about prevalence, diversity and phenotypic or genotypic characteristics of the isolates from the upper respiratory tract may be applied for epidemiological purposes. Recent advances in molecular techniques have generated several typing methods based on PCR for genetic assessment of genetic relatedness of bacterial or fungal strains. One of them, RAPD-PCR (random amplified polymorphic DNA-polymerase chain reaction) analysis is a useful discriminatory method of Candida spp. in clinical practice (Saran et al., 2008; Krawczyk et al., 2009; Bacelo et al., 2010; Gültekin et al., 2011; Samaranayake et al., 2011).

The aim of this study was to estimate by RAPD-PCR the genetic diversity of $C$. albicans isolated from upper respiratory tract of patients with non-small cell lung cancer.

\section{MATERIALS AND METHODS}

The study group of patients. The study enrolled 100 patients with non-small cell lung cancer (NSCLC), hospitalized at the Department of Thoracic Surgery of Medical University of Lublin. The study population consisted of 87 males and 13 females with the average age of 62 years (range 37-73) from South-East Poland. These patients were diagnosed with ca. macrocellularae, ca. planoepithelialae, ca. planoepithelialae partim macrocellularae, ca. macrocellulare partim planoepithelialae and adenocarcinoma. Some patients were after pre- or post-operative chemotherapy including Cis-platina and Vepesid, administered in doses according to the standard procedures. None of the patients had lesions in oral cavity. The Ethical Committee of the Medical University of Lublin approved the study protocol (No. KE0254/75/2011).

Microbiological cultures. Specimens were obtained from throat and nostrils with sterile alginate-tipped

e-mail: ania.biernasiuk@umlub.pl

*These results were presented at the 3rd Workshop on Microbiology in Health and Environmental Protection - Mikrobiot 2013, Łódź, Poland

Abbreviations: RAPD-PCR, random amplified polymorphic DNApolymerase chain reaction 
swabs on aluminium shafts. Within one hour, the swabs were plated on Sabourand agar with chloramphenicol and incubated aerobically at $35-38^{\circ} \mathrm{C}$ for $48 \mathrm{~h}$. Yeast were identified by colony morphology, Gram staining and a biochemical method (API Candida, API AUX BioMerieux).

RAPD-PCR for $\boldsymbol{C}$. albicans differentiation. The DNA from the isolates was prepared using GeneMATRIX Yeast DNA Purification Kit $\left(E R_{\mathrm{X}}\right)$ according to the manufacturer's procedure. RAPD-PCR method was performed with RSD12 primer (Samaranayake et al., 2003; Pinto et al., 2004; Pires-Gonçalves et al., 2007; Waltimo et al., 2001). PCR reactions were carried out in a thermocycler and amplification conditions were as follows: 40 cycles of denaturation at $94^{\circ} \mathrm{C}$ for $30 \mathrm{~s}$, primer annealing at $57^{\circ} \mathrm{C}$ for $2 \mathrm{~min}$ and elongation at $72^{\circ} \mathrm{C}$ for $2 \mathrm{~min}$. The final elongation at $72^{\circ} \mathrm{C}$ was extended to last $15 \mathrm{~min}$. The polymerase chain reactions were performed in $0.5 \mathrm{ml}$ microcentrifuge tubes in a final reaction mixture containing $100-400 \mathrm{ng}$ of $C$. albicans DNA as template, 10xPCR buffer for Taq DNA polymerase (Fermentas), $1 \mathrm{u} / \mu \mathrm{l}$ of Taq DNA polymerase (Fermentas), $200 \mu \mathrm{M}$ dNTPs (Fermentas), $2.5 \mathrm{mM} \mathrm{MgCl}$ (Fermentas), $1.25 \mu \mathrm{M}$ of primer RSD 12 (Proligo primer\&probes).

For each experiment, the size of DNA fragments amplified by PCR was determined by direct comparison with the DNA marker — 100 bp Ladder Plus (Fermentas). Control tubes without template DNA were included in each run and reproducibility was checked for

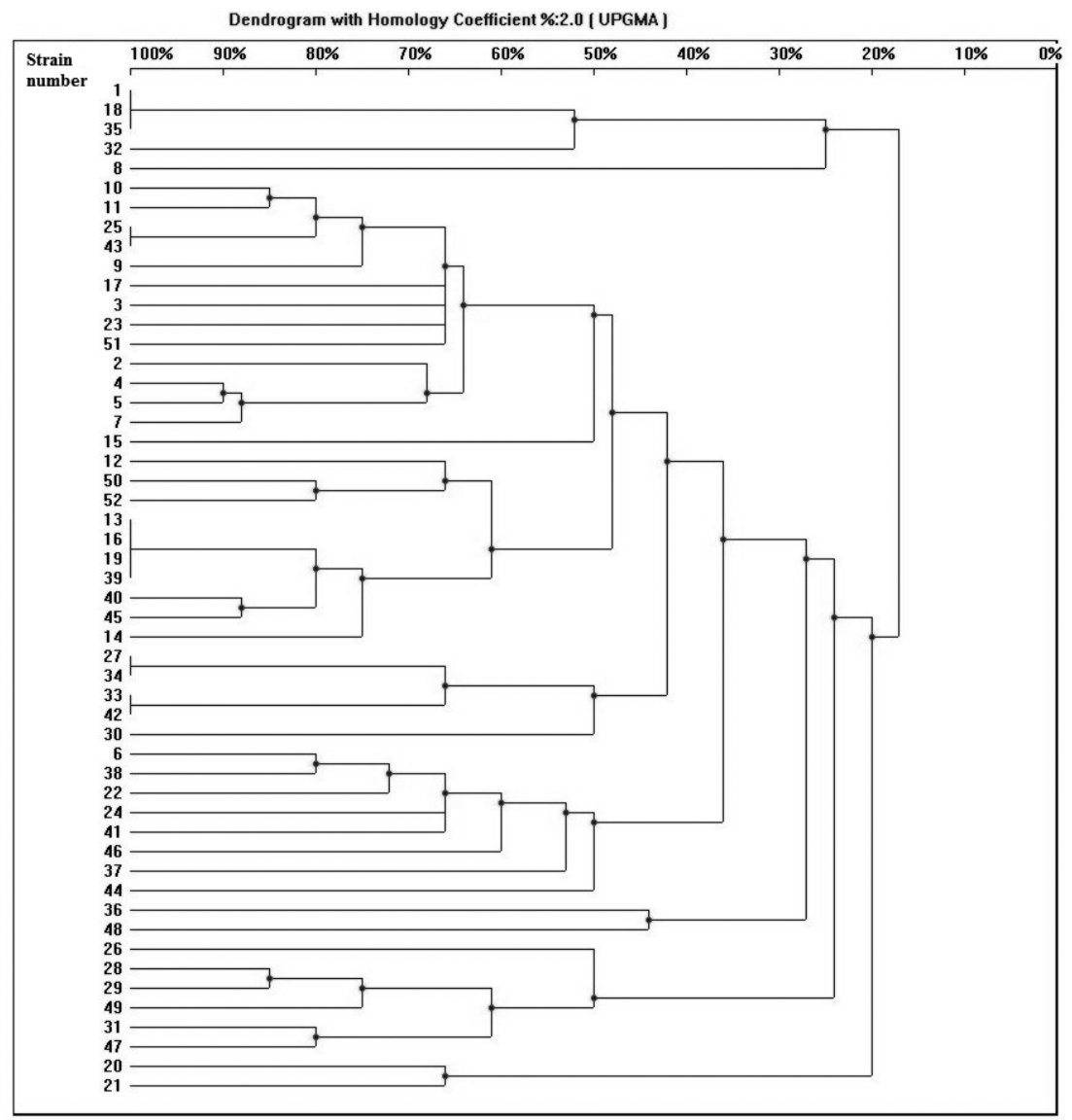

Figure 1. Dendrogram generated for 52 isolates of $C$. albicans from upper respiratory tract of patients with lung cancer on the basis of RAPD-PCR profiles.

Vertical lines mark the positions of the similarity coefficient value $\geq 80 \%$, arbitrarily used as the threshold for clustering of similar genotypes. each reaction. The PCR products were separated using electrophoresis in agarose gels $(1.5 \%)$ at $120 \mathrm{~V}$ for approximately $100 \mathrm{~min}$ at room temperature in TBE buffer (Tris Borate Electrophoretic Buffer, 89 mMTris/ $\mathrm{HCl}, 89$ $\mathrm{mM}$ boric acid, $2.5 \mathrm{mM}$ EDTA, $\mathrm{pH}$ 8.0) (Sigma). Reaction products were detected by ethidium bromide and visualized with UV light.

In silico analysis. Different banding positions of RAPD fingerprinting patterns of $C$. albicans isolates were analyzed using the BioGene program (Polygen). For the analysis of relationships among the strains with this software, dendrograms are constructed by the unweighted pair group method. Each lane pattern was compared to every other pattern through computations of a similarity coefficient, which compares the band positions.

\section{RESULTS}

A total of 52 isolates of $C$. albicans colonizing mucosal membranes of the upper respiratory tract were obtained from NSCLC patients. Phenotypic differentiation was carried out using a biochemical microtest (API Candida, API AUX, BioMerieux). RAPD-PCR technique was used to determine genetic diversity of $C$. albicans isolates. The criteria for genotyping were the differences in banding positions of each isolate in the RAPD-PCR profile. The isolates with identical banding profiles were pooled into a single genotype. Similarity coefficient $\geq 80 \%$ was the value that was arbitrarily used as the threshold for clustering of similar genotypes, since it is roughly halfway between the mean value for dissimilarity and identity. On the basis of RAPDPCR profiles, among 52 strains of C. albicans, 34 genotypes were defined within the overall yeast population, including 10 clusters containing from 2 to 6 isolates, which comprised 28 (53.85\%) strains and unique genotypes represented $24(46.15 \%)$ strains. The coefficient of relatedness of all isolates was 17\% (Fig. 1).

\section{DISCUSSION}

Genotyping by RAPD-PCR technique is easy to perform, versatile, useful and a reliable method allowing for genetic and phylogenetic differentiation of strains belonging to Candida spp., including $C$. albicans, and may be applied as a valuable method for epidemiological purposes (Chong et al., 2003; Samaranayake et al., 2003; Samaranayake et al., 2011). According to data in the literature (Pinto et al., 2004; Noumi et al., 2009; Biernasiuk et al., 2010; Gültekin et al., 2011; Samaranayake et al., 2011), RAPD-PCR is a good method for analyzing the C. albicans genotype, greatly dependent on the choice of primers. Several primers could be used 
in this method, e.g. RSD primers. In our study primer RSD12 was employed. Primers, such as RSD6, RSD10 or RSD11, were also used separately or in combination by other authors (Waltimo et al., 2001; Samaranayake et al., 2003; Teanpaisan et al., 2008; Sun et al., 2009; Szweda et al., 2013).

In our research, primer RSD12 has been successfully used for subgrouping 52 C. albicans strains isolated from the upper respiratory tract in patients with non-small cell lung cancer; similarity coefficient $\geq 80 \%$ was employed, as is usually the case in such studies (Samaranayake et al., 2003; Biernasiuk et al., 2010). The differences in RAPDPCR patterns between the strains revealed their high genetic diversity and occurrence of 34 genotypes.

Our previous studies (Biernasiuk at al., 2010), also with primer RSD12, indicated genetic differences among 75 strains of $C$. albicans isolated from the upper respiratory tract of elderly people, aged of 65 years old or older, staying in close communities, such as a care center; among these strains, 46 genotypes, including 11 clusters containing from 2 to 9 isolates and 36 unique genotypes were found. Similarly, Waltimo and coworkers (2001) using primer RSD12 found some genetic diversity of $C$. albicans isolates from root canal infections in a Finnish population; among the 37 isolates, 10 genotypes were found. Moreover, these authors showed that amplification with primer RSD6 revealed 12 genotypes, while combination of RSD6 and RSD12 indicated the presence of up to 31 genotypes.

In contrast, Sun and coworkers (2009) studying heterogeneity of C. albicans isolated from oral cavity in cancer patients (with hematological malignancies and solid tumors) in China using primer RSD12 obtained quite different results; only 2 genotypes among the 205 strains were found. However, the same authors showed that amplification with primer RSD6 showed 17 genotypes, while the combination of the two primers RSD6 and RSD12 generated 21 genotypes.

The results presented here and some controversial data from the literature (Sun et al., 2009) suggest that genetic diversity assessed by RAPD-PCR of C. albicans strains isolated from the upper respiratory tract or oral cavity, including those with dental infections seems to be dependent not only on the type of primer used but also may be related to origin of the strains from different geographical areas, e.g. Europe or Asia.

\section{CONCLUSION}

The analysis of C. albicans genotypes by RAPD-PCR technique revealed the greatest genetic variability among these isolates compared to phenotypic methods and may be applied as a valuable method for epidemiological purposes.

\section{Acknowledgements}

The results described here were obtained and analyzed with the use of equipment purchased within the agreement No. POPW.01.03.00-06-010/09-00 Operational Program Development of Eastern Poland 2007-2013, Priority Axis I, Modern Economy, Operations 1.3. Innovations Promotion.

\section{REFERENCES}

Bacelo KL, da Costa KR, Ferreira JC, Candido RC (2010) Biotype stability of Candida albicans isolates after culture storage determined by randomly amplified polymorphic DNA and phenotypical methods. Mycoses 53: 468-474.

Biernasiuk A, Korona-Głowniak I, Malm A (2010) Differentiation by RAPD-PCR of Candida albicans isolated from upper respiratory tract in elderly people from care center. Ann UMCS Sec DDD 2: 11-15.

Chong PP, Lee YL, Tan BC (2003) Genetic relatedness of Candida strains isolated from women with vaginal candidiasis in Malaysia. $J$ Med Microbiol 52: 657-666.

Costa F, Manaia CM, Figueiral MH, Pinto E (2008) Genotypic analysis of Candida albicans isolates obtained from removable prosthesis wearers. Lett Appl Microbiol 46: 445-449.

Gültekin B, Eyigör M, Tiryaki Y, Kırdar S, Aydın N (2011) Investigation of antifungal susceptibilities and some virulence factors of Candida strains isolated from blood cultures and genotyping by RAPDPCR. Mikrobiyol Bul 45: 306-317.

Kim J, Sudbery P (2011) Candida albicans, a major human fungal pathogen. J Microbiol 49: 171-177.

Krawczyk B, Leibner-Ciszak J, Mielech A, Nowak M, Kur J (2009) PCR melting profile (PCR MP) - a new tool for differentiation of Candida albicans strains. BMC Infect Dis 9: 177-189.

Lee W, Low BK, Samaranayake LP, Hagg U (2008) Genotypic variation of Candida albicans during orthodontic therapy. Front Biosci 1: 3814-3824.

Noumi E, Snoussi M, Saghrouni F, Ben Said M, Del Castillo L, Valentin E, Bakhrouf A (2009) Molecular typing of clinical Candida strains using random amplified polymorphic DNA and contourclamped homogenous electric fields electrophoresis. I Appl Microbiol 1: 1991-2000.

Pinto PM, Resende MA, Koga-Ito CY, Tendler M (2004) Genetic variability analysis among clinical Candida spp. isolates using random amplified polymorphic DNA. Mem Inst Oswaldo Cruz 99: 147-152.

Pires-Gonçalves RH, Miranda ET, Baeza LC, Matsumoto MT, Zaia JE, Mendes-Giannini MJ (2007) Genetic relatedness of commensal strains of Candida albicans carried in the oral cavity of patients' dental prosthesis users in Brazil. Mycopathologia 164: 255-263.

Samaranayake YH, Samaranayake LP, Dassanayake RS, Yau JY, Tsang WK, Cheung BP, Shiren Y, Issa SY, Badran EF, Akl KF, Shebabi AA (2011) Epidemiological characteristics of Candida species colonizing oral and rectal sites of Jordanian infants. BMC Pediatr 11: 1-6.

Samaranayake YH, Samaranayake LP, Dassanayake RS, Yau JYY, Tsang WK, Cheung, Yeung KWS'(2003) Genotypic shuffling of sequential clones of Candida albicans in HIV-infected individuals with and without symptomatic oral candidiasis. J Med Microbiol 52: 349-359.

Saran B, Karahan ZC, Ağirbaşli H, Tekeli A, Aksoy AM (2008) Comparison of different primers used for the genotyping of Candida albicans clinical isolates by randomly amplified polymorphic DNA method. Mikerobiyol Bul 42: 645-654.

Sun J, Qi Ch, Lafleur M, Qi Q (2009) Fluconazole susceptibility and genotypic heterogeneity of oral Candida albicans colonies from the patients with cancer receiving chemotherapy in China. Int J Oral Sci 1: $156-162$.

Szweda P, Gucwa K, Romanowska E, Dzierżanowska-Fangrat K, Milewski S (2013) Genotyping of clinical isolates of fluconazole-resistant Candida albicans. Proceedings of XIV Conference Diagmol 2013. Molecular biology in diagnostics of infectious diseases and biotechnology 133-135.

Teanpaisan R, Niyombandith M, Pripatnanant P, Sattayasanskul W (2008) Biotypes, genotypes and ketoconazole susceptibility of Candida albicans isolates from a group of Thai AIDS patients. New Microbiol 31: 409-416.

Waltimo TMT, Dassanayake RS, Ørstavik D, Haapasalo MPP, Samaranayake LP (2001) Phenotypes and randomly amplified polymorphic DNA profiles of Candida albicans isolates from root canal infections in a Finnish population. Oral Microbiol Immunol 16: 106-112. 